\title{
AETIOLOGY OF ACUTE PYELONEPHRITIS AND ANTIBACTERIAL RESISTANCE IN CHILDREN WITH AND WITHOUT KNOWN URINARY REFLUX
}

Ieva Kravale ${ }^{1,2}$, Roberts Gobergs 1,3, Elza Salputra ${ }^{4}$, Edīte Jeruma ${ }^{3}$

${ }^{1}$ Department of pathology, Riga Stradiņš University, Latvia

${ }^{2}$ Vidzeme hospital, Latvia

${ }^{3}$ Children Clinical University hospital, Latvia

${ }^{4}$ Jurmala hospital, Latvia

\section{Background and Aims}

Acute pyelonephritis (AP) is common in childhood and around $80 \%$ are caused by E. coli. The choice of empiric therapy should be based on the local antibacterial resistance (AR).

The aim of our study was to determine whether in children with urinary reflux (UR) the aetiological agents and their AR differ from the rest of the paediatric population.

\section{Method}

A retrospective study was performed to determine the differences of aetiological agents and their AR in children with (group A) and without (group B) previously known UR. Medical histories of 400 inpatients with diagnosis of AP or exacerbation of chronic pyelonephritis during the period from January 2015 to December 2016 were selected from archives of tertiary paediatric centre.

\section{Results}

$32 \%(n=127)$ of the patients were male. In $45 \%(n=178)$ the diagnosis was confirmed with a positive urine culture (PUC) and in $7 \%(\mathrm{n}=12)$ of the patients with PUC there was previously known UR (Figure 1). The isolated agents from PUC were E. coli in 50\% $(\mathrm{n}=6)$ and $73 \%(\mathrm{n}=121)$, other single agents in $33 \%(\mathrm{n}=4)$ and $13 \%(\mathrm{n}=22)$, and multiple agents in $17 \%(\mathrm{n}=2)$ and $14 \%(n=23)$ in groups A and B respectively (Figures 2, 3); the difference wasn't significant, $p=0,137$. In 152 PUCs AR to cefuroxime was determined and was higher in the group A $-50 \%(n=5)-$ than in group B $-4 \%(n=6)$ (Figures 4, 5), $\mathrm{p}<0,001$.

\section{Conclusion}

In our study the aetiology of AP didn't differ in patients with and without previously known UR. AR to cefuroxime is significantly higher in patients with known UR and thus should prompt the use of other antibacterial agents in these patients.

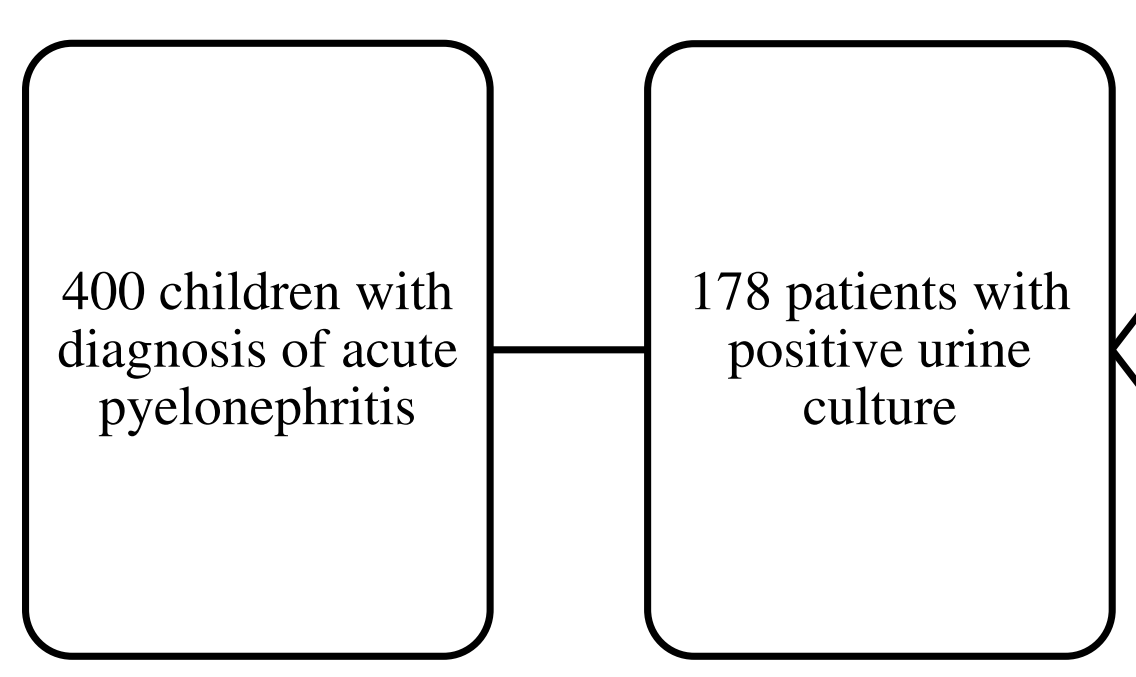

\section{Abbreviations}

$\mathrm{AP}$ - acute pyelonephritis

$\mathrm{AR}$ - antibacterial resistance

PUC - positive urine culture UR - urinary reflux
Figure 2. Isolated agents from positive urinary cultures in children with previously known urinary reflux

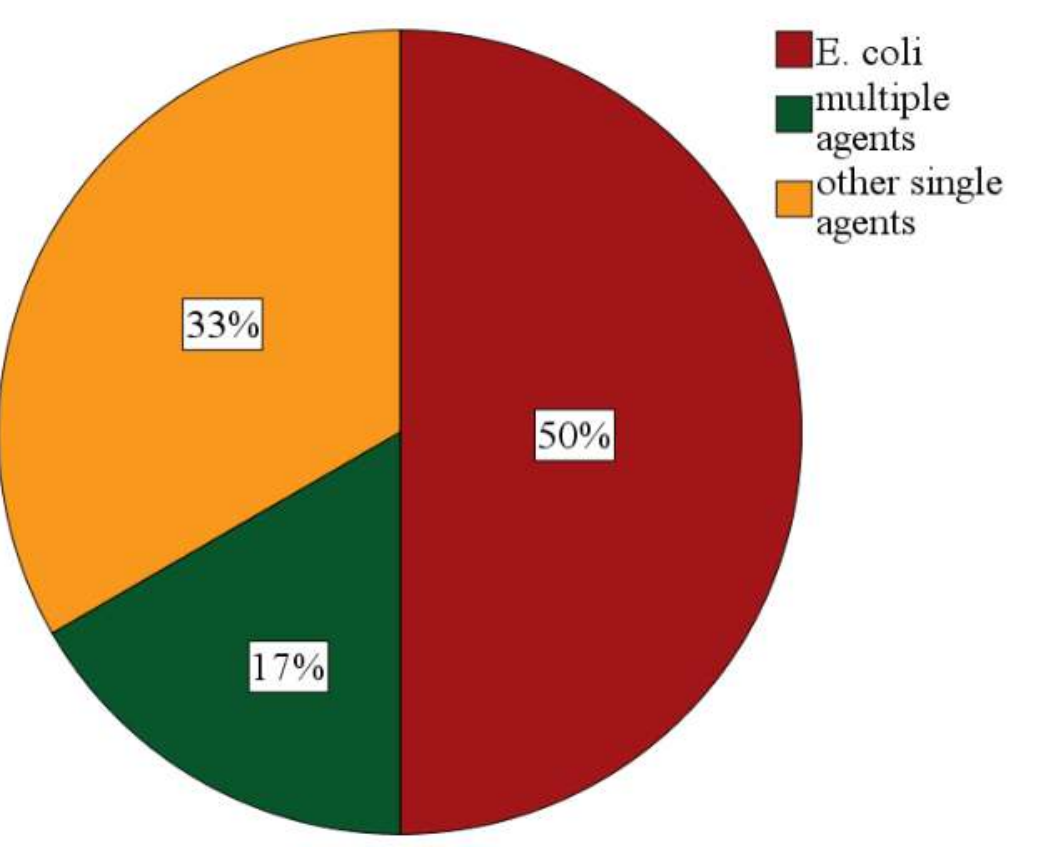

Figure 3. Isolated agents from positive urinary cultures in children without previously known urinary reflux

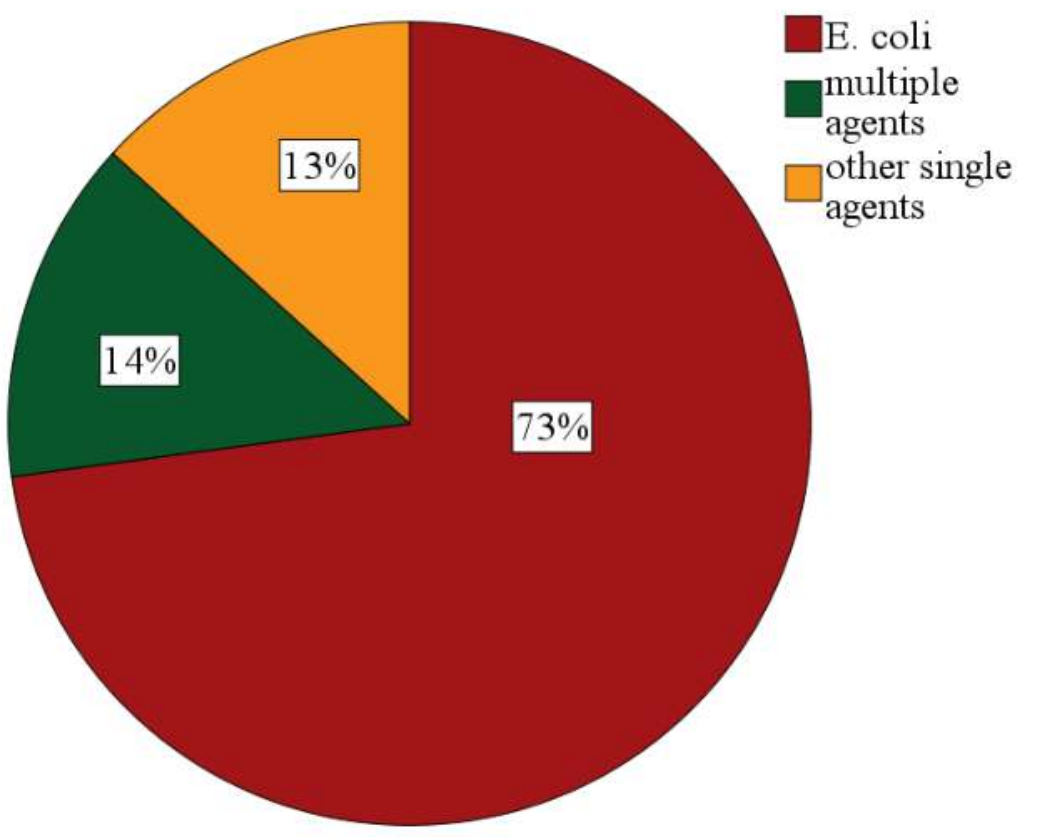

Figure 4. Antibacterial resistance to cefuroxime in children with previously known urinary reflux

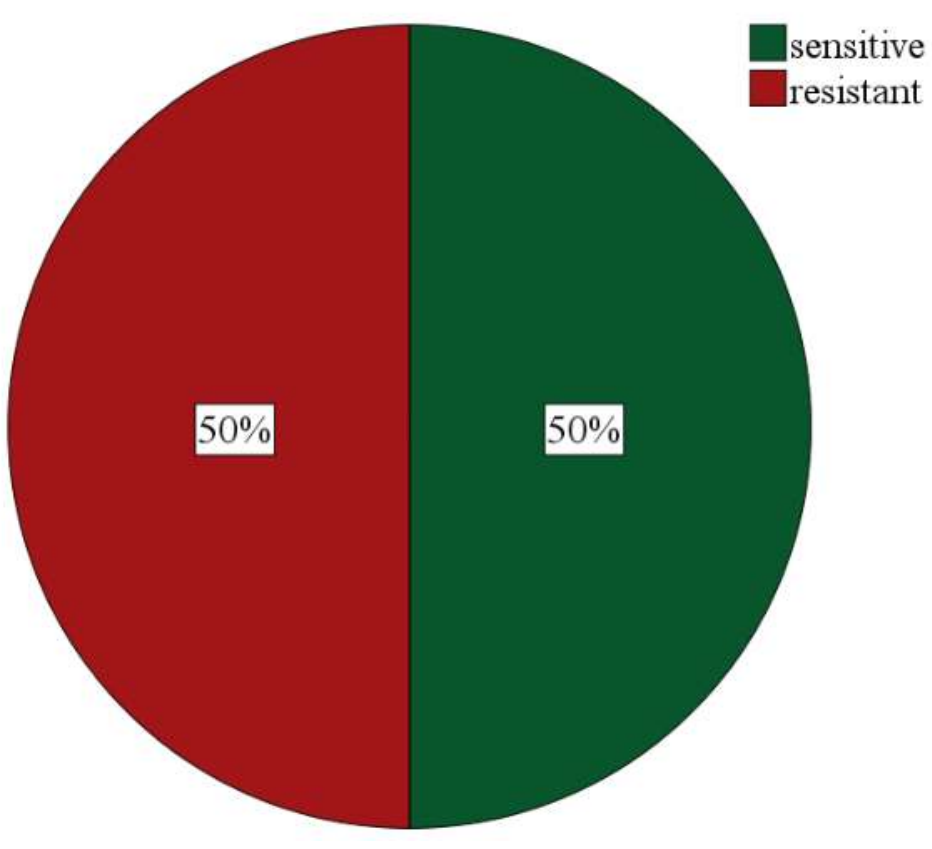

Figure 5. Antibacterial resistance to cefuroxime in children without previously known urinary reflux

142 patients with antibacterial resistance to cefuroxime determined

10 patients with antibacterial resistance to cefuroxime determined

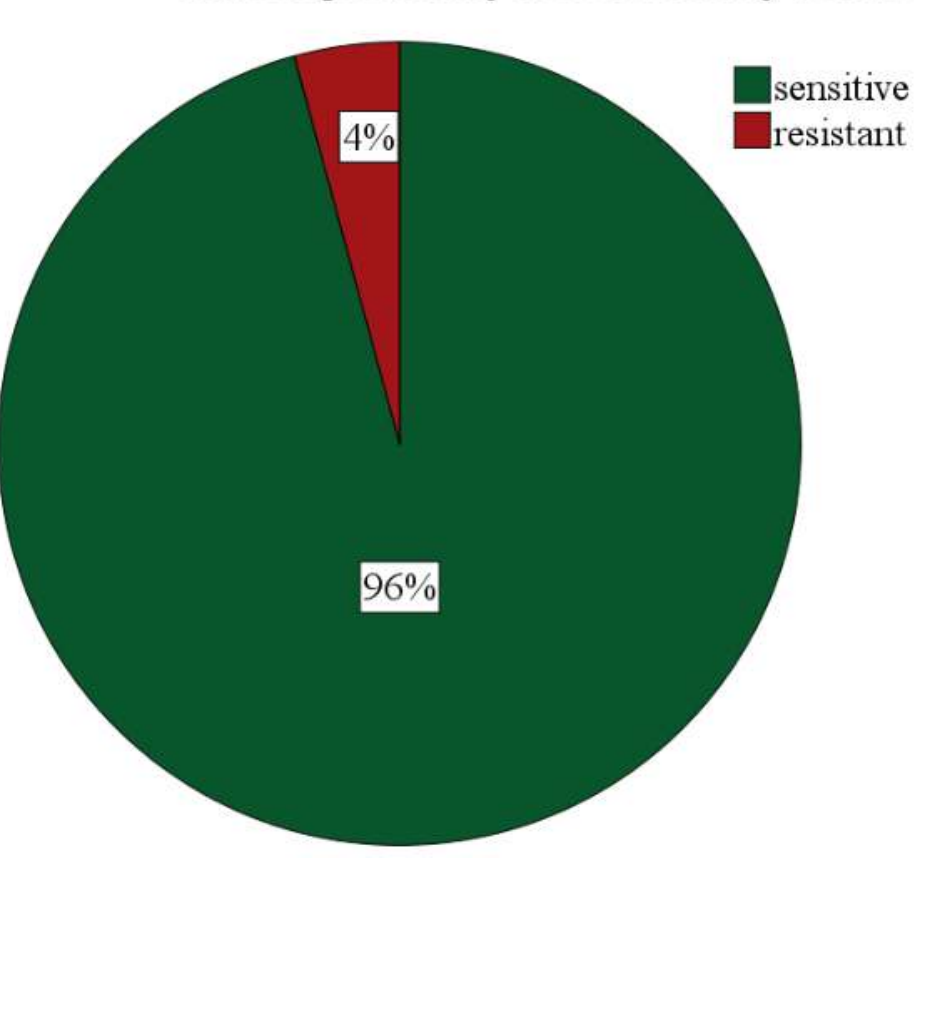

Figure 1. Distribution of analysed cases 